\title{
Feasibility study on walking training biped locomotion surrogate robot
}

\begin{abstract}
This paper discusses the technical feasibility and ethical considerations about walking training of a biped locomotion surrogate robot assisted by a device for reducing the weight load and/or a treadmill, instead of a person with a gait disorder for his safety at the tuning of training period as first ethical condition. For confirming this, some experiments were done using a robot. As the second ethical condition, it was described that the robot was not seen to human. Then, Nebo used here was suitable as a surrogate robot such meanings.
\end{abstract}

Keywords: locomotion surrogate, treadmill, surrogate robot
Volume 2 Issue 5 - 2017

\author{
Makoto Katoh,' Kiichi Katoh,' Yusuke Arita,' \\ Junji Furusho \\ 'Department of Mechanical Engineering, Osaka Institute of \\ Technology, Japan \\ ${ }^{2}$ Fuzzy Logic systems Institute, Japan,
}

Correspondence: Makoto Katoh, Department of Mechanical Engineering, Osaka Institute of Technology, Japan,

Email makoto.kato@oit.ac.jp

Received: July 14, 2017 | Published: August 03, 2017

\begin{abstract}
Abbreviations: MAS, message announcement system; VAS, voice announcement system; QAS, question and answer system; SSS, sound sign system; VOS, voice order system; SOS, sound order system.
\end{abstract}

\section{Introduction}

Over the past few years in Japan, the number of senior citizens has increased remarkably, and around 1,650,000 of them have gait disorders. Gait disorder means to become difficult of gait by many kinds of cause. For examples, cerebrovascular disorders and joint trouble are the main causes of gait disorders, which are common ailments among those aged 65 to 74 . They may be unrelated to the working Conditions. Many researchers have made progress in having robot surrogates replace humans in many dangerous and dirty endeavors, especially in the fields of aerospace ${ }^{1}$ and atomic power, ${ }^{2}$ so as to reduce the need to expose people to risks and also to reduce training costs. However, such robot surrogates have not replaced humans in educational environments. Loco mart like treadmill, wearable power-assistance devices and support robots have been developed for the rehabilitation of people with gait disorders, and load-free treadmills for walking rehabilitation have been executed in a clinical environment. ${ }^{3,4} \mathrm{~A}$ treadmill is a useful apparatus for gait training and for making evaluations; however, many differences have been reported between walking on a treadmill and walking over the ground. Experimental comparisons of the muscular activity of the leg and of the heart rate have been carried out. ${ }^{5}$ The results suggest care be taken because the training adjustment for treadmills may be dangerous and place an excessive load on patients. There are two loads here: The load of the robot receives from a load imposed by an outside force ${ }^{6}$ Many machines and tools have been developed for controlling gait, such as ambulation-support machines ${ }^{7}$ and loco motor training tools. ${ }^{8}$ This research applies Feasibility Study (FS) to the problem of Law Governing Engineering and Engineering Ethics and the necessity of robot control and treadmill control in adjustment of surrogate walking training to reduce the risk of overloading patient. The authors reduce this risk by constructing a two-legged surrogate robot of about $1 / 4$ the scale of an average human being in such an adjustment stage.

\section{Assisted-active walking}

In this section, the authors present the method and the results on 3 kinds of experiments. One is hand-assisted active walking, the other is active walking without hand-assistance, third is a tread-mill assisted active walking with a load reduce device. The authors have not adopted a passive walking using a slope because it is risky for the surrogate robot to a gait disorder patient.

\section{Hand-assisted active walking}

Object of experiments: The load of a person with a gait disorder is evaluated by proxy by quantifying as "light" or "heavy" the motor load on each joint of a surrogate robot imposed by hand-assisted active walking using only an adjustable-load device.

Devices used in the experiments: The Nuevo manufactured by ZMP Co. is used as the two-legged surrogate robot. Nuevo is blue and does not look like a human, even though it is a humanoid robot.

It is normally necessary to use a humanoid robot that is the same size as a human being, and that has the same gait disorder as the patient, to achieve such a technical purpose, that is, evaluating the motor load placed on the joints of a surrogate robot. This paper can be forecast that various ethical problems increase in opposite method of technological engineering ethics. A walking-support apparatus (load device) and a treadmill were manufactures by ourselves, and examined technically easy FS and the ethical problems of using inexpensive ready-made scaled-down robots that do not look human, in order to decrease the development cost and time, and to avoid ethical problems as far as possible.

The authors consider the following conditions for avoiding the mentioned above ethical problem:

a. The color is not human-like even if the figure is like a human.

b. The face is immature, and is not like human but is not lovely either.

c. It is not better than a recent two-legged robot at walking.

The authors consider that the Nuevo is suitable as a surrogate robot for similar second ethical reasons as above. 
Experimental methods: The authors compared various degrees of servo compliance by tuning the motor control gain, namely, the amount of increase in motor torque generated by hand-assistance such as backward movement of the foot in a programmed active-gait state (Figure 1). Figure 2 shows comparison of torch of a joint between with assist and without assist. This experiment was conducted after a load-free state was obtained, using our home-built equipment in which the amount of load on the robot's legs can be varied by tightening or slacking a screw.

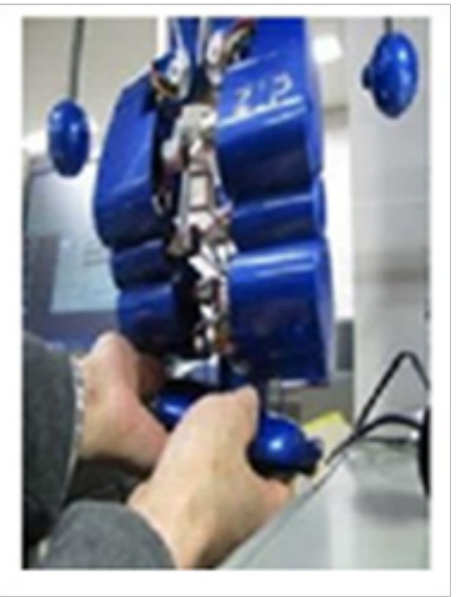

Figure I Hand-assisting.

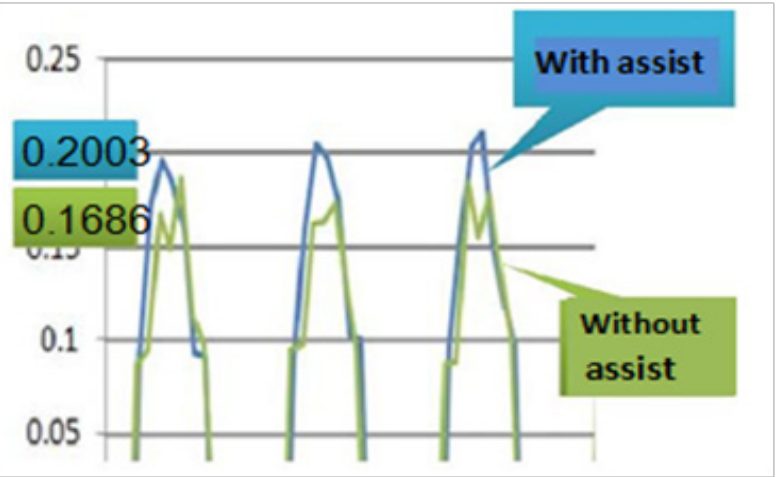

Figure 2 Comparison of tork [N-m] of active walking.

\section{Considerations}

a. That walking is possible in a state where the amount of bending of the joint is decreased in the case of a load-free condition.

b. That the robot can reduce the load to an extent by implementing load reduction using an external force.

c. That the next motion can under taken early because the load on the robot is reduced in the case of completely load-free condition.

d. Those elderly persons can reduce the external load so that they do not place too heavy a load upon their knees.

\section{Treadmill-assisted active walking with a load-reducing device}

There are many studies about treadmill-assisted walking, for example, a weight support tread-mill by center gravity control has been developed for human patient by Furusho et al. ${ }^{9}$ This section shows objects of experiments and devices used in the experiments on simple treadmill-assisted active walking with a load reduction device for testing of the surrogate robot.

Object of experiments: These experiments evaluate the load on a patient is evaluated by proxy as the load imposed on each joint by the treadmill in assisted active walking with the device for reducing the robot load. Table 1 shows the list of major specifications of a tread mill for surrogate walking training tuning.

Table I Shows the list of major specifications of a tread mill for surrogate walking training tuning

\begin{tabular}{ll}
\hline Name of device & Tread mill \\
\hline Width & $370 \mathrm{~mm}$ \\
Depth & $260 \mathrm{~mm}$ \\
Height & $95 \mathrm{~mm}$ \\
Width of Belt & $210 \mathrm{~mm}$ \\
Length of Belt & $670 \mathrm{~mm}$ \\
Power & $12 \mathrm{vBattery}$ \\
MaxValue of Variable Resistance & $42 \Omega$ \\
Range of Speed & $50 \mathrm{~mm} / \mathrm{s} \sim 145 \mathrm{~mm} / \mathrm{s}$ \\
Material & Aluminum, Iron, Rubber, Plastic
\end{tabular}

Experimental methods: These experiments evaluate the torque of each joint motor is evaluated by measuring the current in the motor, and the load for passive walking is evaluated from these motor current readings.

The present study plotted, both the rotation speed of the treadmill and the load were gradually changed and the current in each joint motor. The authors identified regions where the joint load current was within a prescribed range.

Results of experiments: Figure 3 shows the change in torque in the ankle and knee joints when the control gain is adjusted using the treadmill.

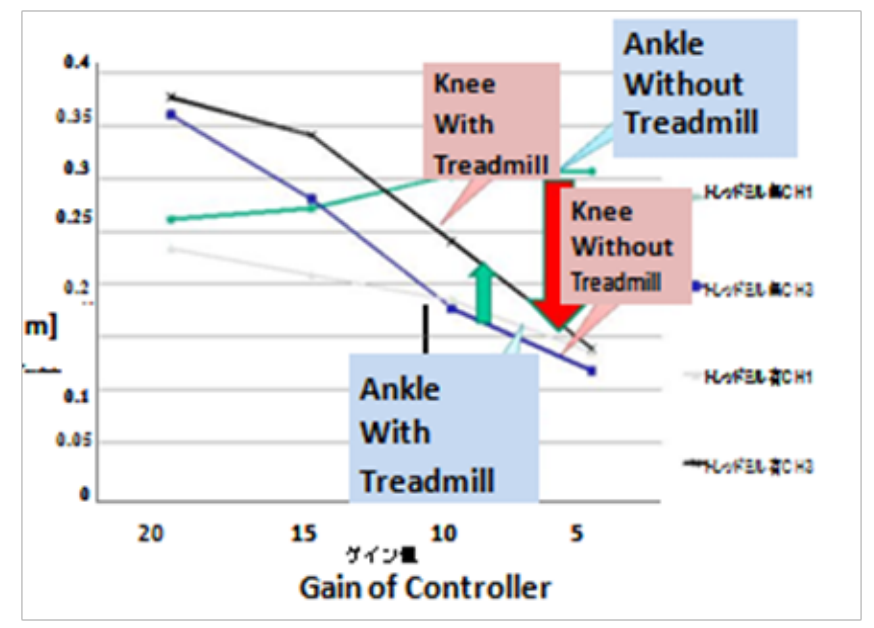

Figure 3 Relation between torque and control gain for each joint motor during treadmill training. 
a. Generally speaking, a hard servo increases the motor torque because the control gain is large-although no large change is seen in this Figure 3. (Here, a hard servo means that the operator feels the servo hard because displacement/force is smaller.

b. The knee torque decreases when the control gain is reduced so that the servo becomes soft (Here, displacement/force is larger). However, there is a tendency for the ankle torque to increase because the robot leg is extended, the leading foot is dragged, and the leading foot catches on the floor.

\section{Considerations}

a. It is expected that the motor torque (the energy for walking) will decrease if the robot is controlled by a hard servo in the fixedleg phase (walking that requires effort), the ankle torque will be decreased in the subsequent phase if it is controlled by a soft servo in the moving-leg phase (walking in which force is weakened, and its knee torque is lowered.

b. It is expected that human- like walking can be achieved by using gain scheduling control that synchronizes the walking phase (repeated fixed leg and moving leg).

\section{Future plans}

Figure 2 shows the limitations of this work and details concerning the surrogate robot and the training apparatus, as well as the directions for future achievement are shown in Table 2. There is a room for improvement because the number of degrees of freedom arrangement of the leg of the surrogate robot used in this research is considerably different from that of a human. It might be difficult for the surrogate robot like this to express abnormal long time fixed pain etc. by the artificial face expression. ${ }^{10,11}$

Table 2 Future development policy for robot and devices

\begin{tabular}{|c|c|c|c|}
\hline & \multicolumn{2}{|l|}{ Future development policy } & \multirow[b]{2}{*}{ Achievement method } \\
\hline & Future requirement & This study & \\
\hline \multirow[t]{2}{*}{ Robot } & Human-like walking & Constant gain & $\begin{array}{l}\text { Variable gain (gain Scheduling according } \\
\text { to state) }\end{array}$ \\
\hline & Human-like driving method & Motor control & Artificial muscle \\
\hline \multirow{2}{*}{ Device for training } & $\begin{array}{l}\text { Automatic speed tuning of } \\
\text { treadmill }\end{array}$ & Constant speed & Detection of walking speed by sensors \\
\hline & $\begin{array}{l}\text { Independent load tuning of each } \\
\text { joint }\end{array}$ & Overall load tuning & Device for load tuning of each joint \\
\hline
\end{tabular}

\section{Discussion and considerations}

The three robot principles of Asimov are famous as a basis for robot design, and the concept is mainly based on defending human life and not harming it. The authors considered also the following requirements for surrogate training robots, including the reasons for choosing the Nuevo as a surrogate:

a. The walking training of a genuine patient must not test for until the safety can confirm though an enough condition cannot be set yet here.

b. 'The pain that sees the robot suffer like human is not given to spectators', summarizing, 'the robot is not seen to human' though it is likely to reverse as shown later according to purpose.

It is expected that the result of this research will be useful in the promotion of surrogate robot development (although there is a room for more discussion as to whether a humanoid robot would be more suitable) for the adjustment of rehabilitation training apparatus. There are more interesting themes in this area, that is, the message announcement system (MAS), voice announcement system (VAS), question and answer system (QAS) ${ }^{12}$ and the sound sign system (SSS) by the surrogate robot, the voice order system (VOS) and the sound order system (SOS) $)^{13}$ by the human or robot instructor, etc.. Moreover, any text announcement, order and sign using the concept of the possession phenomena like ITACO from PC display to the robot display, ${ }^{14}$ and/or monitoring of any sign by artificial face expression (pain etc. ${ }^{10,11}$ ) for remote control of treadmill robot $^{15}$ by instructors will be future interesting themes.

\section{Conclusion}

First, this paper presents hand assisted active walking and treadmill assisted active walking with a load reducing device using simple non-human like robot Nuevo as walking training surrogate robot with consideration and future plan. Moreover, this paper discusses and considerations about requirements for surrogate training robots referring to three principles for robot design of Asimov, and more interesting themes in the future.

\section{Acknowledgment}

We wish to express our gratitude to Lecturer Hokuto Hakogi of the Toin Yokohama University for their leading study on rehabilitation, Lecturer Tomi Hiroi of the Robotics Department who shared valuable research results about the psychological impact of a robot's external appearance, and Lecturer Shun Ushida of the Mechanical Engineering Department for valuable discussions on the compliance of servos and visual feedback systems.

\section{Conflict of interest}

Finally, our thanks go to many students, especially Mr. Junnichi Sawaki, Mr. Masaki Ishitani and Ms Natsuki Imura, and many teachers who cooperated in this study and paper at our laboratory and university. 


\section{References}

1. Rosheim ME. Robotic Surrogate Work in Progress. Proc IEEE International Conference Robot Automation.1996(1):396-403.

2. MC Scully, NJ Porter, JW Scott. Use of Surrogate travel to lower training costs and reduce person-rems. 1985. p. 1-4.

3. Nakazawa K. Attempt of damage spinal cord function maximization by Crai walking standing to training. Journal of Spine spinal cord. 2004;17(11): 1035-1041.

4. Shimizu Y, Suzuki R, Eiichi SAITOH, et al. A Preliminary Report about a new Robot WPAL (Wearable Power-Assist Locomotor) for Paraplegic Gait Reconstruction. Jpn Journal of Rehabilitation Med. 2009;46(8):527-533.

5. Nagata, Yamamoto M, Funabiki S. Gait Evaluation of Over ground Walking and Treadmill Walking Using Compass-Type Walking Model. IEEJ Transactions on Electronics, Information and Systems. 2009;129(7):1212-1219.

6. Michimen K, Ohsu R. Advancement and development of rehabilitation medicine Feed forward movement training of Hen'asa lamplight arm. Present age medical treatment. 2000;32(6):1445-1450.

7. Kai Y, Inoue Y. A Study of Control Methods of an Ambulation Support Machine: A Study on the basis of Leg Muscular Tensions while Pacient's falling. Proceedings of Japan Robot Associates Conference. 2002;20:1L16.
8. Nakazawa K, Akai M. On possibility of gait orthoses as a locomotor training tool. Bulletin of the Japanese Society of Prosthetic and Orthotic Education, Research and Development. 2005;21(3):125-130.

9. Ozawa TT, Nisio D, Furusho J, et al. Development of Weight Support Treadmill with the Center Gravity Control. Experimental Kinematic. 2010;10(2):156-161.

10. Lu G, Li X, Li H. Facial Expression Recognition for Neonatal Pain Assessment. IEEE Int Conf Neural Networks \& Signal Processing. 2008. p. $456-460$.

11. Monwar MM, Rezaei S. Video Analysis for View-Based Painful Expression Recognition. IJCNN. 2008. p. 3619-3626.

12. Katoh M, Imura N, Washio K. Imperative improvement from failure mode effect analysis to action mode effect, inference, and yield analysis of miss-over-orders. SICE. 2017.

13. Inoue T. Life support for elderly using a communication partner robot: Speech technology, useful or not for assistive technologies? Welfare Information Technology (WIT). 2011;111(226):75.

14. Ono T. Toward Realization of Ambient Intelligence on the basis of Human Agent Intelligence. IPSJ SIG Reports. 2009;155(3):13-16.

15. Sakamoto D, Kanda T, Ono T. Tele-operation interface of communication robots based on switching between different camera views. IPSJ SIG Reports. 2006;145(7):37-42. 